\title{
Adaptive Crisp Active Contour Method for Segmentation and Reconstruction of 3D Lung Structures
}

\author{
Pedro Pedrosa Rebouças Filho \\ Federal Institute of Technology of Ceará \\ Av. Treze de Maio, 2081 \\ Benfica, Zip-code: 60040-531
}

\author{
Roger Moura Sarmento \\ Federal Institute of Technology of Ceará \\ Av. Treze de Maio, 2081 \\ Benfica, Zip-code: 60040-531
}

\author{
Paulo C. Cortez \\ Federal University of Ceará \\ Campus do Pici, S/N
}

\author{
Antônio Carlos da Silva Barros \\ Universidade de Fortaleza \\ Washington Soares Av. 1321, B1. J-30 \\ Edson Queiroz, Zip-code: 60811-905
}

\author{
Victor Hugo C. de Albuquerque \\ Universidade de Fortaleza \\ Washington Soares Av. 1321, B1. J-30 \\ Edson Queiroz, Zip-code: 60811-905
}

\begin{abstract}
Computing systems have been playing an important role in various medical fields, notably in image diagnosis. Highlighted among the existing exams that allow diagnostic aids and the application of computing systems in parallel is Computed Tomography (CT). This work focuses on the segmentation and reconstruction phases of CT lung images using the Adaptive Crisp Active Contour Model 2D (ACACM) and the OpenGL library to present and analyse the results in three dimensions. The results of the proposed method were compared with those of the 3D Region Growing method and then evaluated by two pulmonologists. The results showed the superiority of the proposed method, thus confirming that that this method could integrate medical diagnostic aid systems in the pulmonology field. Finally, some applications are shown utiizando segmentation and 3D reconstruction proposals demonstrating that the proposed method can be used to aid in medical diagnosis.
\end{abstract}

\section{General Terms:}

Lung Structures, Computed Tomography, Computer Vision

\section{Keywords:}

Crisp Active Contour Method, Computed Tomography, Image Segmentation, 3D Reconstruction, Lung Structures.

\section{INTRODUCTION}

A large number of diseases that affect the population of the world are lung-related. Therefore, research in the field of Pulmonology has great importance in public health studies and focuses mainly on asthma, bronchiectasis and Chronic Obstructive Pulmonary Disease (COPD) [33, 15].

The World Health Organization (WHO) estimates that there are 300 million people who suffer from asthma, and this disease causes around 250 thousand deaths per year worldwide [4]. Also, WHO estimates that 210 million people have COPD. This disease caused the death of over 300 thousand people in 2005 [32]. Recent studies reveal that COPD is present in the 20 to 45 year-old age bracket, although it is characterized as an over-50-year-old disease. WHO estimates that the number of deaths due to COPD will increase $30 \%$ by 2015 , and by 2030 COPD will be the third cause of mortality worldwide [9].

In the period from 1992 to $2006,15 \%$ of all hospitalizations financed by the Brazilian Federal Health System (SUS) were due to pulmonary diseases, of which asthma and COPD together summed up 562,016 hospitalizations [4].

Thus, it is of fundamental importance for the public health system to obtain an early and correct diagnosis of any pulmonary disease. Diagnostic aid is important from a clinical point of view as it increases the amount of information the specialist has concerning the patients state of health. Therefore, with such data certain illnesses can be detected precociously, and lives saved lives in some cases. Also, some techniques allow the clinical image of the disease to be tracked appropriately [11, 21].

The segmentation stage of CT pulmonology images is essential for the correct and accurate medical diagnosis, as this stage delimits the lung area in CT images of the thorax, which must be analyzed by the diagnostic aid or by a specialist.

The segmentation of objects and structures in medical images is a process that, in most cases, is more complex if compared to the segmentation of other image types, such as metallographic and synthetic aperture radar remote sensing. This is due to the variability of structures and/or internal organs, and the different visualization planes of these images. Furthermore, there is the possibility of diseases affecting these organs. All of which contribute to increase the difficulty to develop efficient techniques for medical image segmentation [11, 21].

Lung segmentation techniques have been developed to optimize this step. Among these techniques, [30] and [11] used the region growing method to obtain lung segmentations of CT images. [12], [17] and [29] put together Mathematical Morphology, Region 
Growing, Thresholding and Edge Detection methods to obtain even more accurate results. [7] e [19] used traditional Active Contour Model (ACM) techniques for lung segmentation; meanwhile [27] used the Watershed transform.

Another methodology for lung segmentation is the 3D Region Growing approach, and this is applied to segment the lung and its internal structures, such as the vessels and airways [3, 18, 31, 22]. This method works similarly to the $2 \mathrm{D}$ region growing technique that is initialized by a seed point and then expanded through its neighbors, obeying an aggregation rule.

However, the traditional threshold-based segmentation methods, and the Region Growing, Edge Detection and Watershed methods do not normally present consistent results in CT thorax images [14, 24]. This may happen due to the lack of precision in the detection of lung edges generated by the internal structures such as veins, arteries and airways [8] or due to pulmonary disease or noise which can distort the radiological densities expressed in HU (Hounsfield units) of the lungs [14]. Also, segmentation techniques that utilize edge information or region characteristics separately, in general, present insufficient results and are only adequate for specific cases [14]. This is because these techniques often include structures that do not constitute the pulmonary tissue, such as the hilum and parts of the thorax cavity [17, 28, 29, 10] which are not under analysis. In order to overcome the technical restrictions of the previously mentioned methods, other segmentation techniques have been developed. The most important ones are the Pulmonary Emphysema Detection System (PEDS) [13], the Active Crisp Contour Method [24] and the Active 2D Hilbert Contour method [14]. The PEDS system automatically segment the lung using the Region Growing technique associated with morphological closing and erosion operations [13].

In this work, the Adaptive Crisp Active Contour two-dimensional Method (ACACM) was used for lung segmentation [24]. This method has shown itself to be superior compared to other CT image lung segmentation methods, in efficiency, as well as efficacy. This work aimed to segment a whole lung in a CT thorax exam, segmenting each image individually through ACACM, and used the result of each piece of the CT exam to generate a three dimensional (3D) lung segmentation. The results were compared to the 3D Region Growing method results, which is the most widely used method for this type of work found in the literature. Then, both methods were evaluated by two physicians (pulmonologists).

These methods aim to assist medical diagnostic aids in pulmonology diseases using 3D visualization and reconstruction from two-dimensional CT images. After image segmentation it is possible to visualize and quantify the lung structures, as well as their internal structures three-dimensionally. This facilitates the diagnosis by the medical experts and reduces/eliminates the subjectivity of interpretation of the exam. The results obtained from the proposed method and the 3D Region Growing method were evaluated and validated by two medical specialties (physicians/pulmonologists). One of the important contributions of this work is to use the proposed method on personal computers (PC), maintaining, or in some cases improving the performance. Also a PC reduces the processing costs, the use of the CT scanner workstation as well as reducing the overall time to evaluate a CT image.

\section{EXPERIMENTAL PROCEDURES}

This work proposes a new segmentation and 3D lung reconstruction method. The Adaptive Crisp Active Contour Method (ACACM) was used to segment the lung from each part of the CT thorax image, and a new methodology using the open-source computer graphics library (OpenGL) was proposed to reconstruct the lung and its respective $3 \mathrm{D}$ visualization.

\subsection{Medical Image Acquisition}

The tomographic models used to acquire the complete exams were the Toshiba Aquilion (TA), the GE Medical system LightSpeed16 (GEMSL) and the Phillips Brilliance 10 (PB). All images had a 512 x 512 resolution with 16 bits. Table 1 gives the characteristics of these exams, conceded by a pulmonologist, with the patients authorization. These images constitute an image cluster obtained in partnership with the Walter Cantdio Hospital of the Federal University of Cear, submitted to an earlier study [33, 15]. This study was approved and evaluated by the UFC Research Ethics Committee COMEPE, (Protocol n 35/06) and complied with the requirements of Resolution n 196/96 of the National Health Council, concerning research in human beings [33 15.

Table 1. Description of the exams used to analyze the 2D and 3D algorithms.

\begin{tabular}{c|c|c|c|c}
\hline $\begin{array}{c}\text { Number of } \\
\text { exam }\end{array}$ & $\begin{array}{c}\text { Number of } \\
\text { images }\end{array}$ & $\begin{array}{c}\text { Slice } \\
\text { thickness }\end{array}$ & $\begin{array}{c}\text { Tomographic } \\
\text { model }\end{array}$ & $\begin{array}{c}\text { Kind of } \\
\text { Pathology }\end{array}$ \\
\hline \hline 1 & 908 & $0.5 \mathrm{~mm}$ & TA & Normal \\
2 & 297 & $0.5 \mathrm{~mm}$ & TA & Normal \\
3 & 685 & $0.5 \mathrm{~mm}$ & TA & Normal \\
4 & 760 & $0.5 \mathrm{~mm}$ & TA & Normal \\
5 & 229 & $3.0 \mathrm{~mm}$ & TA & COPD \\
6 & 278 & $1.25 \mathrm{~mm}$ & GEMSB & Normal \\
7 & 267 & $1.25 \mathrm{~mm}$ & GEMSB & Fibrosis \\
8 & 239 & $1.25 \mathrm{~mm}$ & GEMSB & Normal \\
9 & 276 & $2.0 \mathrm{~mm}$ & PB & Fibrosis \\
10 & 296 & $2.0 \mathrm{~mm}$ & PB & Normal \\
11 & 597 & $1.0 \mathrm{~mm}$ & PB & COPD \\
\hline
\end{tabular}

The CT lung images shown in Table 1 were acquired on apex, hilum and on the base of the axial plane, under the following conditions: Each slice was $1.5 \mathrm{~mm}$ thick, field of vision was $312 \mathrm{~mm}$, electrical tension in the tube was $120 \mathrm{kV}$, electric current in the tube was $200 \mathrm{~mA}$, lung window adjustment: centre and width, were 600 and $1600 \mathrm{HU}$ respectively, the dimension of the reconstructed window was $512 \times 512$ pixels and the voxels had dimensions of $0,585 \times 0,585 \times 1,5 \mathrm{~mm}$ and were quantified in 16 bits.

\subsection{Automatic CT image lung segmentation using ACACM}

The ACACM method [24] appeared as an evolution of the Crisp Active Contour Method [26] for thorax CT image lung segmentations. The ACACM can identify the edges of objects with concavities, projections or bifurcations without restrictions. Therefore the total energy at a point $c(s)$ of ACACM is given by [24]:

$$
E_{C R A D}[c(s)]=E_{\text {int }_{\text {adap }}}[c(s)]+E_{\text {ext }_{C R A D}}[c(s)],
$$

where $E_{\text {int adap }}$ is the internal adaptive energy and $E_{\text {ext }} t_{C R A D}$ is the external Crisp Adaptive energy.

The internal proposed ACACM 2D energy is given by [24]:

$$
E_{\text {int }_{\text {adap }}}[c(s)]=w_{\text {cont }} F_{\text {cont }}[c(s)]+w_{\text {adap }} F_{\text {adap }}[c(s)],
$$

where $F_{\text {cont }}[c(s)]$ is the same Continuity force used in the traditional ACM proposed by [20] and $F_{a d a p}[c(s)]$ is the new Balloon Adaptive force. The parameters $w_{\text {cont }}$ and $w_{\text {adap }}$ are the weights 
associated with each force to adjust the importance of each one of its respective terms in the calculation of the internal energy $E_{\text {int }}$ adap .

The Balloon Adaptive force uses topology information of the curve to expand the contour. This information is specific for each point, and assumes that the curve possess distinct formats along its topology. In this way, the Adaptive Balloon force dislocates each point of the curve towards the edges of the object of interest, adapting the topology it is placed onto.

To calculate this new internal force, information based on the coordinates of the point of interest of the immediate neighbours and the information based on the form of the curve is obtained. The data of the coordinates of the point of interest of the immediate neighbours is used to calculat $F_{\text {adap }}$. These coordinates are used to determine the mean point of the neighbours, which is used as reference to determine the direction of $F_{a d a p}$. The other information is given by the location analysis of this mean point, determining if it is inside or outside the curve.

After finding this topology data $F_{a d a p}$ is given by [25]:

$$
F_{\text {adap }}[c(s)]=\sqrt{E_{x}^{2}+E_{y}^{2}},
$$

where $E_{x}$ and $E_{y}$ components are defined by:

$$
E_{x}=\left|x(s) \pm x_{m}\right|,
$$

and

$$
E_{y}=\left|y(s) \pm y_{m}\right|,
$$

where $x(s)$ and $y(s)$ are the coordinates of the point $c(s)$ where $F_{\text {adap }}$ is being calculated, $x_{m}$ and $y_{m}$ are the coordinates of the neighbours mean point. The topology information concerning location determines the plus or minus sign used in Equations 4 and 5 . negative means the point is external to the curve and positive when the point is internal.

Concerning the external energy of the ACACM method, the proposed $E_{\text {ext }}$ CRAD uses the concept of Lung Densities Analysis (LDA) of a neighbourhood applied to the Crisp ACM method [24]. This is done to obtain the percentages of the $6 u_{i}$ classes, in which $i$ varies from 0 to 5 :

$-u_{0}$ - Hyper aerated ( -1000 to $\left.-950 \mathrm{HU}\right)$;

$-u_{1}$ - Normally aerated ( -950 to $\left.-500 \mathrm{HU}\right)$;

$-u_{2}$ - Poorly aerated ( -500 to $\left.-100 \mathrm{HU}\right)$;

$-u_{3}$ - Not aerated ( -100 to $\left.100 \mathrm{HU}\right)$;

$-u_{4}$ - Bone (600 to $\left.2000 \mathrm{HU}\right)$;

$-u_{5}$ - Areas not classified (densities that do not fit with the others).

To bypass the external Crisp energy restrictions, this new energy incorporates a multilayer perceptron artificial neural network (MLP) to determine the origin of each edge found in the thorax CT images. The inputs of this MLP are the 6 percentages of the $u_{i}$ classes found by the LDA method [26]. Meanwhile there is only one output to indicate that the edges found in thorax images are or are not pulmonary walls.

In this method just one hidden layer is used in the topology of MLP is used, and this layer is responsible for solving non linearly separable problems. Thus, to demonstrate the quantity of neurons in the hidden layer (HN) the solution described by [34] was used:

$$
H N=\frac{2 \times \max \left(M_{1}, M_{2}\right)}{3},
$$

in wich $M_{1}$ and $M_{2}$ are the quantities of neurons in the input and output, respectively.
The result of Equation 6 uses $M_{1}$ as the 6 percentages of the $u_{i}$ classes and $M_{2}$ as the only output to determine if the edge belongs to the lung or not. This results in a $6 / 4 / 1$ configuration, in other words, 6 entry neurons, 4 neurons in the occult layer and 1 neuron in the output. The specified topology is verified by the method of cross-validation [16], in which a conjunction of samples is separated randomly, and one group is used for the training of the neural network and the other for experimental tests, in order to validate the classifier.

The training data were extracted from thorax CT images in the apex, hilum and base positions of patients with COPD, fibrosis and healthy volunteers as described in Section 2.1

The data for the training phase are selected manually in order to obtain a greater representation of different pulmonary structures. On average, 150 pixels were added per image, indicating whether the origin of the edges found by the Sobel operator were from the pulmonary wall or were made up of the bone edges, veins airways, air and other structures. In this training step 500 images from different exams, diseases and levels of cuts were used.

The data of the input group was obtained from a $P$ pixel $\left(x_{p}, y_{p}\right)$, where $x_{p}$ and $y_{p}$ are the coordinates of the pixel used in the function $f\left(x_{p}, y_{p}, s_{i}\right)$, to obtain all $u_{i}$ percentages used as input of MLP. The training of the neural network is carried out and tested according to the cross-validation [16].

Using the previously trained MLP, the execution phase of its output is given by the function:

$f_{m l p}(\mathbf{u})=\left\{\begin{array}{cc}1, & \text { when the detected edge constitutes a pulmonary wall and } \\ 0, & \text { otherwise. }\end{array}\right.$

Using $f_{m l p}$ to determine the origin of the edges found in thorax CT images, the external energy $E_{e x t}{ }_{C R A D}$, defined in Equation 1 . is given by:

$$
E_{\text {ext }_{C R A D}}(x, y)=\left\{\begin{array}{l}
S(x, y), \text { para } f_{m l p}(\mathbf{u})=1 \text { and } \\
1, \text { otherwise, }
\end{array}\right.
$$

where $S(x, y)$ is the Sobel operator, and $\mathbf{u}$ is the percentage vector of the LDA method [26], using the coordinates $(x, y)$ of the analyzed pixel.

The external energy of this method is capable of identifying the origin of the edges of fibrosis, emphysema and healthy lungs. This is because MLP has been trained to recognize these patterns, as they are the more common cases seen on lungs in thorax CT images. The MLP adopted by this work was retrained inserting fibrosis that was not used by [24], in thus increasing the scope of applications for this method.

The automatic segmentation of the lungs in thorax CT images uses the automated curve initialization, the addition and the point removal methods are described in ACACM [24].

The first step to automatically segment the lung in CT images is to open the DICOM image using the open-source library DCMTK. After that, the external force of the image is calculated using the LDA method and a MLP to detect the origin of the edges obtained by the Sobel operator. The edges detected within the lung are excluded from the external energy, however they are used as the initialization of the curve.

The curve is initialized using the proposed method, in which the false lung edges determine the point of initialization within the lung. This point is a regular polygon centroid used as the initialization of the curve.

This curve moves by successive iterations of the three method sequence: ACACM minimizing its energy moving the points of the 
curve, angle-based point removal and point addition in the curve. In the flowchart this method sequence is called cycles, and the letter $n$ indicates the order of the results obtained in each $n$ cycle.

The curve is labelled stable when the perimeter does not increase after two successive iterations. When this happens, lung segmentation is concluded, and the generated polygon is said to be the segmentation result of the object of interest.

\subsection{D visualization}

Application Programming Interface (API) Open Graphics Library was used for the $3 \mathrm{D}$ visualization. This API is an open specification and multiplatform of a modelling and graphical routine library used for computer graphic applications, such as games and visualization systems [2].

OpenGL is extremely efficient and many of its algorithms are installed in modern graphic hardware [1]. It operates in a similar way to a $\mathrm{C}$ library, and provides a number of functions. Normally a program is based on OpenGL or it is an OpenGL application, which means that it is written in some programming language that calls one or more OpenGL libraries. The OpenGL applications vary from $\mathrm{CAD}$ tools to modelling programs used to create cinema characters, such as a dinosaur [1].

Besides basic graphics, such as lines and polygons, OpenGL supports illumination, colouring, texture mapping, transparency and animation, among other special effects. Nowadays, OpenGL is recognized and accepted as an API standard for the development of real time graphical 3D applications [5].

This work used two libraries from OpenGL, GLU and GLUT, both open source. GLU (OpenGL Utility Library) contains numerous routines that uses low level OpenGL commands to execute tasks such as, for an example, define the matrixes for the projection and orientation of the visualization, and rendering of a surface [5]. GLUT (OpenGL Utility Toolkit) is a platform with an independent toolkit that includes graphical interface elements [1].

Thus, the $3 \mathrm{D}$ visualization system in this work was developed in $\mathrm{C} / \mathrm{C}++$ language, and call routines from the OpenGL library. GLU library is called to render objects, configure their looks and their illumination. The GLUT library, which is a multiplatform visualization system, is used to create windows and receive user commands. To model the 3D objects the data structure was assumed to be a stacked block of $2 \mathrm{D}$ data and since the 2D data are images then the 3D data were considered to be a block of piled images. These images have a space between them, which depends on each application.

In the specific case of CT imaging, the spacing between images, or planes, is one of the properties stored in the DICOM pattern, defined by the Slice Thickness property. The size of a pixel is also specific for each application when these images are modelled in $3 \mathrm{D}$, and in the DICOM pattern, which is determined by the Pixel Spacing property. Using these two parameters it is possible to reconstruct a CT exam on the same scale that it was generated.

Each plane represents an image, so each plane can have a curve, represented by a determined polygon. As previously seen, these curves are composed of points and are connected with their neighbours. Figure 1(a) illustrates the data structure used in this model, where each plane possesses a curve composed of interconnected points. In this Figure the points are presented in red, and the connections between them in green.

To render an object in $3 \mathrm{D}$, the points that belong to each plane must be connected to the other layers, and not just to the neighbouring points of their original plane, as in the data structure of the ACACM method. Thus each point of a layer is connected to the two nearest

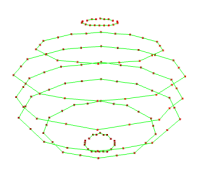

(a)

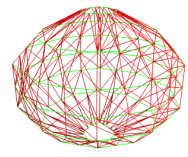

(b)

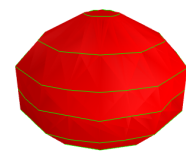

(c)
Fig. 1. Rendering stages a) overlapping of the existing curves in each image; b) connection of different layers generating triangles; and c) rendered surface.

points of the layer immediately superior to it. This operation generates a net, linking all the layers through traced lines between them. Figure 1(b) presents an example of these connections, in which the lines between points of the same layer are in green, and the lines between points of different layers are in red.

To complete the rendering proposed in this work, the net presented in Figure 1(b) is used to generate the surface of the 3D object. These surfaces are generated by triangles, in which two neighbouring points of a layer and the closest point to these in a superior layer compose each triangle. A sample of an object generated this way is shown in Figure 1(c)

Properties such as colour, lighting, texture, including the surface transparency can be configured using the OpenGL library.

Therefore the $3 \mathrm{D}$ visualization to render objects uses three distinct stages: overlap the curves presented in each image, connect the points of different layers to build triangles and generate surfaces from these triangles.

\section{EXPERIMENTAL TESTS AND DISCUSSIONS}

In this work, the Adaptive Crisp Active Contour Model is compared with the 3D Region Growing method for the segmentation of the lung in CT exams. A total of 11 complete CT thorax exams of healthy, fibrosis and COPD patients were used.

The configuration used in the ACACM is adapted from [24], using the parameters $\alpha=0.6, \beta=0.1$ and $\tau=0.3$ in the composition of the total energy. Figure 2 shows a step-by-step example of the segmentation carried out by this method from the initialization in Figure 2(a) to the stabilization in Figure 2(f) It is important to mention that each image of a slice of the CT exam possess the initialization of its contour independently of the other slices. Each contour seeks its own stabilization independently according to the flowchart in Figure ??. Figure 2 presents the evolution of the contour group through $3 \mathrm{D}$ visualization proposed in this article.

The 3D Region Growing (RG) method uses the same methodology proposed by [23], using the same initialization as in the ACACM method applied in three dimensions, while the adopted neighbouring region addition method uses information from the anatomy of the lung, by adding voxels that are in some intensity bracket inside the lung: normally aerated, poorly aerated or hyper aerated. This addition takes place by successive iterations, until stabilization is reached, when no voxel is added.

The 3D RG method was chosen because it is automatic form of the radiologist makes the scanner for the lungs. The method is semiautomatic utiizado by the radiologist, he will click on regions of interest and delimiting the regions of interest by expanding the click of your region through neighbors that are in the range of predefined radiographic density as aerated regions, little aerated, etc. When this operation is performed manually take approximately 40 


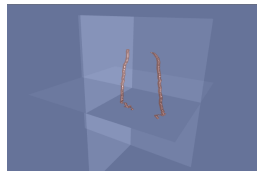

(a)

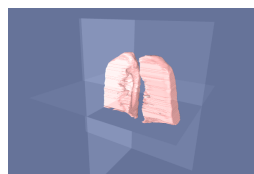

(d)

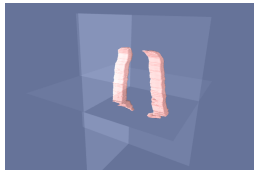

(b)

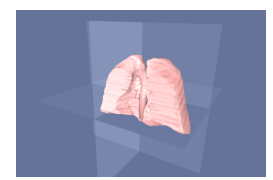

(e)

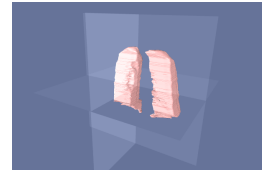

(c)

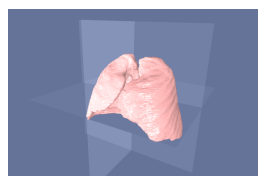

(f)
Fig. 2. Example of the evolution of the ACACM method for the lung segmentation of thorax CT exams: a) initialization b) to c) evolution of the method and f) final result.

minutes. Thus, this automatic implementation of 3D RG makes the fastest method, which makes a lot of authors use this methodology in medical image processing.

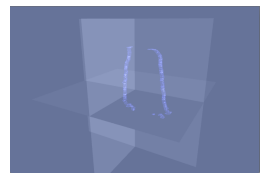

(a)

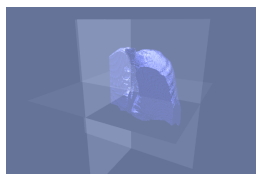

(d)

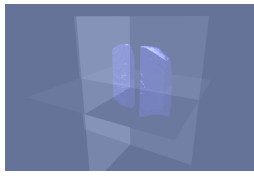

(b)

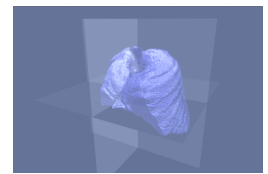

(e)

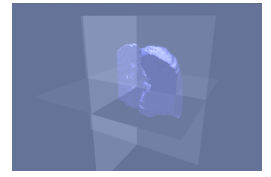

(c)

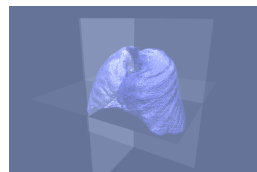

(f)
Fig. 3. Example of the evolution of the 3D RG method for the lung segmentation of CT thorax exams: a) initialization b) and c) evolution of the method and d) final result.

The results of the 3D segmentation visualization using ACACM for each slice are shown in pink, following the visualization structure proposed in Section 2.3 using only the 3D contour of the object to build the visualization. The 3D Region Growing segmentation is presented in blue and uses a solid construction of the whole region obtained by this method. Therefore, some of the lighting and shadowing effects are not the same as the results obtained by the ACM Adaptive Crisp 3D. The construction prevents such effects when using the graphical library OpenGL as in this work.

The segmentation of structures other than the lung, such as the trachea, is common with the 3D RG method, as well as the exclusion of pulmonary parts that present diseases such as fibrosis. Thus, to evaluate the results obtained by these two methods, a qualitative evaluation made by two specialists and an evaluation of the processing time to measure the cost benefit of each method is necessary.

\subsection{Qualitative evaluation by pulmonology physicians}

The segmentation of the 3D method is performed using a complete thorax CT exam, in these kinds of exams the number of images can vary from one procedure to another, depending on the patients body structure and the thickness of the slice of the DICOM image. Thus, since thorax CT exams vary from 250 to 1000 images, an evaluation of all images by a pulmonologist physician becomes impracticable. Thus, a sample of the images to be analyzed is necessary.

The set of images for analysis was made up of 3 images for each CT exam region, totalling 12 images per exam. As 11 complete exams were used, a total of 132 images were employed in the evaluation by specialized physicians for the lung segmentation in thorax CT exams, corresponding to 264 lungs evaluations. This was accomplished using qualitative metrics due to the impossibility of using quantitative ones, as it would be impracticable for a specialist physician to manually segment a total of 132 images, which is overly dull, tedious and tiresome.

Therefore a qualitative evaluation was used for the lung segmentation assessment of the 3D Region Growing and ACACM methods. This evaluation consisted of attributing grades to the segmentations of the two methods by the two pulmonologist physicians responsible. The grades attributed have values ranging from 1 to 5 :

(1) Worst, no part of the object of interest is found;

(2) Bad, just a small part of the object of interest is obtained;

(3) Reasonable, medium quality, with some serious errors;

(4) Good, with few errors;

(5) Best, high quality, as good as is required.

The physicians that assessed the results of the said methods are professors and pulmonologists at the Walter Cantdio University Hospital of the Federal University of Cear (UFC), and are nominated physician 1 (M1) and physician 2 (M2).

The assessment of the physicians here was used to build the Contingency Table, also called confusion matrix, for the evaluation of each method. The lines present the assessments of the physician M1 and the columns those of physician M2, for all the 264 lungs analyzed. The contingency tables of the physicians evaluation of 3D Region Growing and the ACACM 2D are shown in Tables 2 and 3 , respectively.

Table 2. Qualitative assessment by physicians M1 and M2 for the results obtained by $3 \mathrm{D}$ RG.

\begin{tabular}{|c|c|c|c|c|c|c|cc|}
\cline { 2 - 9 } \multicolumn{1}{c|}{} & \multicolumn{10}{c|}{ M2 } \\
\hline & Grade & 1 & 2 & 3 & 4 & 5 & Total(M1) & {$[\%]$} \\
\hline & 1 & 0 & 0 & 0 & 0 & 0 & 0 & $(0)$ \\
\hline & 2 & 0 & 5 & 10 & 5 & 0 & 20 & $(7.58)$ \\
\cline { 2 - 10 } & 3 & 0 & 1 & 62 & 60 & 0 & 123 & $(46.59)$ \\
\cline { 2 - 9 } & 4 & 0 & 0 & 6 & 61 & 9 & 76 & $(28.78)$ \\
\cline { 2 - 9 } & 5 & 0 & 0 & 0 & 6 & 39 & 45 & $(17.05)$ \\
\cline { 2 - 9 } & Total(M2) & 0 & 6 & 78 & 132 & 48 & 264 & $(100)$ \\
& {$[\%]$} & $(0)$ & $(2.27)$ & $(29.55)$ & $(50)$ & $(18.18)$ & $(100)$ & \\
\hline
\end{tabular}

Based on the presented contingency tables it is possible to measure the inter observer concordance, using the kappa index (K) [6]. Thus the value of Kappa obtained through the data in Table 2 is 0.464 , indicating a moderate agreement between the physicians for the assessment of the 3D Region Growing method.

But for the results obtained by ACACM, the Kappa value obtained through the data in Table 3 is 0.614 , indicating a substantial agreement between the physicians.

For a better analysis of the results, Table 4 presents a summary of the percentages obtained by the 3D RG and ACACM methods in the assessments by the physicians, extracted from Tables 2 and 3 . respectively. 
Table 3. Qualitative assessment by physicians M1 and M2 for the results obtained by ACACM.

\begin{tabular}{|c|c|c|c|c|c|c|cc|}
\hline \multicolumn{10}{c|}{} & \multicolumn{10}{c|}{ M2 } \\
\hline Grade & 1 & 2 & 3 & 4 & 5 & Total(M1) & {$[\%]$} \\
\hline 1 & 0 & 0 & 0 & 0 & 0 & 0 & $(0)$ \\
\hline 2 & 0 & 1 & 0 & 0 & 0 & 1 & $(0.38)$ \\
\hline \multirow{4}{*}{ M1 } & 3 & 0 & 0 & 1 & 2 & 1 & 4 & $(1.51)$ \\
\cline { 2 - 9 } & 4 & 0 & 0 & 4 & 201 & 11 & 216 & $(81.82)$ \\
\cline { 2 - 8 } & 5 & 0 & 0 & 0 & 13 & 30 & 43 & $(16.29)$ \\
\cline { 2 - 8 } & Total(M2) & 0 & 1 & 5 & 216 & 42 & 264 & $(100)$ \\
& {$[\%]$} & $(0)$ & $(0.38)$ & $(1.89)$ & $(81.82)$ & $(15.9)$ & $(100)$ & \\
\hline
\end{tabular}

Table 4. Qualitative assessment by physicians M1 and M2 for the results obtained by the 3D RG and ACACM methods.

\begin{tabular}{c|c|c|c|c}
\hline \hline \multirow{2}{*}{$\begin{array}{c}\text { Classification } \\
\text { method }\end{array}$} & \multicolumn{2}{|c|}{ 3D RG } & \multicolumn{2}{c}{ ACACM } \\
\cline { 2 - 5 } & M1 [\%] & M2 [\%] & M1 [\%] & M2 [\%] \\
\hline \hline 1 & 0.00 & 0.00 & 0.00 & 0.00 \\
2 & 7.58 & 2.27 & 0.38 & 0.38 \\
3 & 46.59 & 29.55 & 1.51 & 1.89 \\
4 & 28.78 & 50.00 & 81.80 & 81.82 \\
5 & 17.05 & 18.18 & 16.29 & 15.90 \\
\hline \hline Satisfactory & 45.83 & 68.18 & 98.11 & 97.72 \\
\hline \hline
\end{tabular}

The percentage indexes shown in Table 4 for physician agreement show that the assessment results of the 3D RG method are divergent. This can be seen in the classifications 3 and 4 . In the classification 3 the physician M1 has $46.59 \%$ and physician M2 29.55\%, meanwhile in classification 4, the physician M1 has $28.78 \%$ and physician M2 50\%. This analysis presents the disagreement between the evaluating physicians, and is shown by the Kappa index of 0.464 , indicating moderate agreement between them.

The same analysis for the ACACM results concerning the agreement between the physicians, and using the percentage index obtained from Table 4, shows that the assessment is convergent, and the difference between the physicians is less than $1 \%$ for all classification levels. This fact is supported by the Kappa index of 0.614 , indicating a substantial agreement between them.

However, analyzing the indexes obtained from Table 4 concerning the quality of the segmentation results indicates that the two methods for classes 1 and 5 are close. They do not have incidence of class 1 because no doctor assign the worst rating for the result. Meanwhile, the results on class 5, the percentages obtained by the 3D RG method were $17.05 \%$ and $18.18 \%$, a little higher than the ones obtained by ACACM that were $16.29 \%$ and $15.9 \%$, however, the results for classes 2, 3 and 4 are distinct between these methods. In class 4 , the results obtained by the $3 \mathrm{D}$ RG were $28.78 \%$ and $50 \%$, indicating that physician M1 observed few mistakes in $28.78 \%$ and physician M2 identified $50 \%$ in this method. However, both physicians obtain the same $81.82 \%$ for ACACM, indicating an acceptable class (few errors) in more than $80 \%$ of the images.

Considering the serious errors determined by class 3 , the results obtained by 3D RG were $46.59 \%$ and $29.55 \%$ by ACACM were $1.51 \%$ and $1.89 \%$, indicating that for ACACM the presence of serious errors was less than $2 \%$ according to both physicians. This does not occur for the 3D RG method, where physician M1 identifies the classification reasonable (serious errors) in $46.59 \%$ and physician M2 29.55\%.

While for class 2, the results obtained by $3 \mathrm{D}$ RG were $7.58 \%$ and $2.27 \%$ and for ACACM they were $0.38 \%$ and $0.38 \%$, indicating that both physicians identified a bad segmentation, as just a small part of the object of interest was obtained by both methods. The incidence for 3D RG was more significant as it achieved $7.58 \%$, in just one image while ACACM produced a percentage index of $0.38 \%$.

Considering the general segmentation quality, two of the classes, class 4 and class 5 were considered satisfactory. These two classes are considered satisfactory because they indicate that the segmentations did not possess any flaws or only minor errors, and therefore the physicians can use the results to analyze the internal regions as they are similar to the ideal region or they are the region of interest itself. Besides, any minor errors can be corrected manually. Thus, satisfactory results are obtained by adding classes 4 and 5 .

Following this the 3D RG method obtained $45.83 \%$ and $68.18 \%$, while the ACACM 2D method obtained $98.11 \%$ and $97.72 \%$ of satisfactory results according to the physicians M1 and M2, respectively. Thus the ACACM applied to the 3D segmentation of the lung in thorax CT images is superior to the 3D RG according to the two pulmonology physicians. The ACACM model had only $1.89 \%$ and $2.28 \%$ of unsatisfactory results.

Figures 4 and 5 show some example results of these methods presented by the physicians. One example in Figure 4 shows two distinct classifications presented by the physicians, due to the presence of the hilum and the trachea as pulmonary regions. Physician M1 considered this a serious error and physician M2 considered it a minor error.

Thus in Figure 4 for the 3D RG method physician M1 attributed grade 3 to the lung on the left of the Figure 4(b) due to the presence of the trachea and on both lungs of Figure 4(e) due to the presence of the hilum; however M1 attributed grade 5 to the lung on the right of Figure 4(b). For the ACACM method, physician M1 assessed the segmentation results of Figure 4(c) with grade 5 and the results shown in Figure 4(f) with grade 4. The analysis of physician M2 was the same as physician M1, except for the results with the presence of the hilum or trachea to which he attributed grade 4 as this physician consider this to be a minor error.

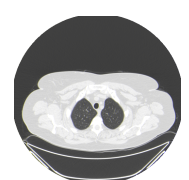

(a)

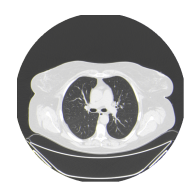

(d)

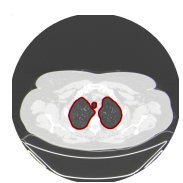

(b)

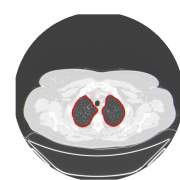

(c)
Fig. 4. Example of the lung segmentation in CT images, a) and d) original images. The hilum is aggregated to the pulmonary region by the $3 \mathrm{~d} R \mathrm{R}$ as seen in b) and e), which does not happen with ACACM; the ACACM results are shown in c), and f).

Other errors in the lung segmentation of the CT images that the physicians disagreed upon are shown in Figure 5 in which, in addition to the error generated by the presence of the hilum or trachea, there was also a lung disease, in this case fibrosis.

In Figure 5 the physicians agreed with the assessment that the disease existed. Thus for 3D RG they attributed grade 3 to both lungs presented in Figure 5(b) due to the lack of the fibrosis regions in the 


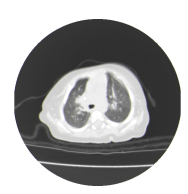

(a)

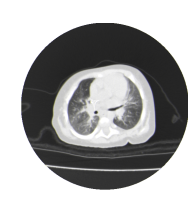

(d)

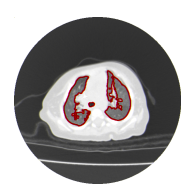

(b)

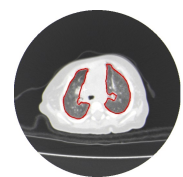

(c)

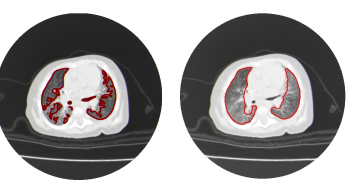

(e)

(f)
Fig. 5. Example of the lung with fibrosis segmentation in CT images, a) and d) original images. The fibrosis region is excluded from the pulmonary region by the $3 \mathrm{D}$ RG as seen in b) and e), which does not happen with ACACM; the ACACM results are shown in c), and f).

segmentation, and considered this to be a serious error, as well as the fact that the lung on the left also aggregated the trachea into its region. Also, the physicians assessed the segmentation of the lung on the right of Figure 5(e) with grade 3 as the fibrosis regions were not in the segmentation and this was considered a serious error; and the lung on the left was given grade 2 as only part of the object was segmented because fibrosis was present in the greater part of the lung. For the ACACM 2D method the physicians attribute grade 4 to the lungs on the right of Figures 5(b) and 5(e) but also noted the presence of minor errors and grade 3 to the lungs on the left of the same Figures, due to the presence of some serious errors.

\section{CONCLUSION AND FUTURE WORKS}

This work proposed a new segmentation methodology for the lungs in thorax CT exams, using a 2D segmentation method and the OpenGL library for a 3D visualization and reconstruction.

The 2D segmentation method used is called Adaptive Crisp Active Contours Model that is able to segment both healthy lungs, lungs suffering from emphysema, and lungs with fibrosis. This latter disease is harder to segment as the diseased pulmonary tissue is similar to healthy pulmonary tissue. This extra ability of this method is possible because the MLP existing in the calculation of the external energy of the ACACM method that defines the origin of the pulmonary edges can be adjusted.

The other main contribution of this article is the new methodology of reconstruction and visualization of lungs in 3D using the OpenGL library. The combination of this methodology with the ACACM method generated a new 3D automated segmentation method of lungs in thorax CT images.

The new methodology of 3D segmentation of the lung is compared with the 3D RG method in the segmentation of thorax CT exams of healthy volunteers and patients with COPD and fibrosis. The results were assessed in a qualitative form by two pulmonologist physicians. According to the physicians M1 and M2 3D RG had $45.83 \%$ and $68.18 \%$, while the proposed method had $98.11 \%$ and $97.72 \%$ of satisfactory results, respectively. This shows that the proposed method is superior to 3D RG according to both pulmonology physicians, and that ACACM has only $1.89 \%$ and $2.28 \%$ of unsatisfactory results. Thus the physicians M1 and M2 concluded that the proposed method was 2 and 1.5 times better than the 3D Region Growing method, respectively.
The proposed method was also better than the 3D RG method in terms of physician agreement for each method. The proposed method received a substantial concordance, while the 3D RG just had a moderate concordance. This was because the 3D RG method presented errors, such as the hilum and trachea in the pulmonary region, exclusion of part of the lung with disease, and the joining of lungs, among another errors, whereas the proposed method did not. These errors were evaluated in different ways by the two physicians, generating a lower agreement than the proposed method. However, the 3D RG method was superior in the processing time by spending half the processing time of the proposed method. This work and the results do not bring an end to this line of research. Future works applying other techniques of computational intelligence and pattern recognition to detect lung edges and to investigate and developed methods to recognize disease in lungs or other organs, as well as to adapt the developed methods to detect the pulmonary lobes are still required.

\section{Acknowledgements}

The authors acknowledge the financial support and encouragement from CNPq.

The authors thank the Post-Graduate Program in Computer Science from IFCE and Pulmonology and Image of Walter Cantdio University Hospital, Federal University of Cear.

\section{REFERENCES}

[1] Dave Astle and Kevin Hawkins. More OpenGl Game Programming. Thomson, EUA, 2005.

[2] Dave Astle and Kevin Hawkins. Begnning OpenGl Game Programming. Thomson, EUA, 2nd edition, 2009.

[3] S. Born, Dirkiwamaru, M. Pfeile, and D. Bartz. 3-step segmentation of the lower airways with advanced leakagecontrol. IJCAI 2009 Workshop on Explanation Aware Computing, pages 239-255, 2009.

[4] H.S. Campos and A.C.M.A. Lemos. Asthma and copd in view of the pulmonologist. Brazilian Journal of Pulmonology, 35(4):301-309, 2009.

[5] Marcelo Cohen and Isabel Harb Manssour. OpenGl: Uma abordagem prática e objetiva. Novatec, 2006.

[6] R. G. Congalton and K. Green. Assessing the accuracy of remotely sensed data: Principles and practices. Lewis Publishers, New York, 1999.

[7] José Silvestre Serra da Silva. Lung segmentation on studies CT scanner. Master's thesis, Department of Electronics and Telecommunications, University of Aveiro, 2005.

[8] Tarique da Silveira Cavalcante. Technique for automatic segmentation of airway in computed tomography images of the chest. Master's thesis, Universidade Federal do Ceará, Fortaleza, Ceará, Brasil, 2010.

[9] R. de Marco, S. Accordini, I. Cerveri, A. Corsico, J. Sunyer, F. Neukirch, N. Kunzly, B. Leynaert, C. Janson, T. Gislason, P. Vermeire, C. Svanes, J.M. Anto, and P. Burney. An international survey of chronic obstrutive pulmonary disease in young adults according to gold stages. Thorax, 59:120-125, Julho 2004.

[10] J. H. S. Felix, P. C. Cortez, and M. A. Holanda. A new algorithm for automatic segmentation of lung CT images. pages 1-6, Campos do Jordão, SP, 2008. XI Congresso Brasileiro de informática em Saúde - CBIS, Sociedade brasileira de informática em saúde. 
[11] J. H. S. Felix, P. C. Cortez, M. A. Holanda, and R. C. S. Costa. Automatic segmentation and measurement of the lungs in healthy persons and in patients with chronic obstructive pulmonary disease in ct images. volume 18, pages 370-373, Margarita Island, Venezuela, Outubro 2007. V Latin American Congress on Biomedical Engineering 2007, Bioengineering Solutions for Latin America Health.

[12] John Heber S. Felix, P. C. Cortez, M. A. Holanda, D. F. Colaço, V.H. C. Albuquerque, and A. R. Alexandria. Lung and chest wall structures segmentation in CT images. pages 291-294. Computational Vision and Medical Image Processing (VIPMAGE), Outubro 2007.

[13] John Hebert Silva Felix, Paulo César Cortez, Pedro Pedrosa RebouçasFilho, Auzuir Ripardo de Alexandria, Rodrigo Carvalho Sousa Costa, and Marcelo Alcantara Holanda. Identification and quantification of pulmonary emphysema through pseudocolors. Lecture Notes in Computer Science, 5317:957964, 2008

[14] John Hebert Silva Flix. Methods of Active Contours in Hilbert $2 D$ image segmentation of lungs in CT of the Chest. PhD thesis, Department of Computer Engineering, Federal University of Cear, Fortaleza, Ceará, Brasil, 2011.

[15] Simone Castelo Branco Fortaleza. Acute effects of administration of continuous positive airway pressure mode noninvasive about airing of lung parenchyma in patients with chronic obstructive pulmonary disease. Master's thesis, Department of Clinical Medicine, Federal University of Cear, Fortaleza, CE, 2006.

[16] Simon Haykin. Neural Networks: A Comprehensive Foundation. Prentice-Hall, EUA, 2nd edition, 1999.

[17] Shiying Hu, Eric A. Hoffman, and Joseph M. Reinhardt. Automatic lung segmentation for accurate quantitation of volumetric X-ray CT images. IEEE Transactions on Medical Imaging, 20:490-498, Junho 2001.

[18] B. Irving, P. TaylorR, and A. Todd-Pokropek. 3D segmentation of the airway tree using a morphology based method. $I J$ CAI 2009 Workshop on Explanation Aware Computing, pages 297-307, 2009.

[19] Yoshinori Itai, Hyoungseop Kim, and Seiji Ishikawa. A segmentation method of lung areas by using snakes and automatic detection of abnormal shadow on the areas. International Journal of Innovative Computing Information and Control, 3:277-284, Abril 2007.

[20] Michael Kass, Andrew Witkin, and Demetri Terzopoulos. Snakes: Active contour models. International Journal of Computer Vision, 1:321-331, 1987.

[21] Tan Kok Liang, Toshiyuki Tanaka, Hidetoshi Nakamura, Toru Shirahata, and Hiroaki Sugiura. An automated 3D emphysema extraction method using lung CT. SICE Annual Conference 2008, pages 3110-3114, 2008.

[22] S. Matsuoka, T. Yamashiro, G.R. Washko, and Y. Kurihara. Quantitative CT assessment of chronic obstructive pulmonary disease. RadioGraphics, 30:55-66, 2010.

[23] P. P. Rebouças Filho, P. C. Cortez, and V. H. C. Albuquerque. 3D segmentation and visualization of lung and its structures using CT images of the thorax. Journal of Biomedical Science and Engineering, 6:1099-1108, 2013.

[24] P. P. Rebouças Filho, P. C. Cortez, J. H. S. Felix, Tarique da Silveira T. S. Cavalcante, and M. A. Holanda. Adaptive 2D crisp active contour model applied to lung segmentation in CT images of the thorax of healthy volunteers and patients with pulmonary emphysema,. Brazilian Journal of Biomedical Engineering, 29:363-376, 2013.

[25] Pedro Pedrosa Rebouças Filho, Paulo Cesar Cortez, Antonio Carlos da Silva Barros, and Victor Hugo Costa de Albuquerque. Novel adaptive balloon active contour method based on internal force for image segmentation - a systematic evaluation on synthetic and real images. Expert Systems with Applications, pages 7707-7721, 2014.

[26] Pedro Pedrosa Rebouças Filho, Paulo César Cortez, and Marcelo Alcantara Holanda. Active contour modes crisp: new technique for segmentation the lungs in CT images. Brazilian Journal of Biomedical Engineering, 27:259-272, 2011.

[27] Rushin Shojaii, Javad Alirezaie, and Paul Babyn. Automatic lung segmentation in CT images using watershed transform. IEEE International Conference on Image Processing-ICIP, 2:1270-1273, Setembro 2005.

[28] A. Silva, J. S. Silva, B. S. Santos, and C. Ferreira. Fast pulmonary contour extraction in X-ray CT images: A methodology and quality assessment. volume 4321, pages 216-224, San Diego, EUA, 2001. Proceding SPIE Conference on Medical Imaging: Physiology and Function from Multidimensional Images, SPIE Digital Library.

[29] Aristófane C. Silva, Paulo Cezar P. Carvalho, Rodolfo A. Nunes, and Marcelo Gattass. Segmentation and reconstruction of the pulmonary parenchyma. pages 73-82. VI Workshop de Informática Médica - WIM, Maio 2006.

[30] Ingrid Sluimer, Mathias Prokop, and Bram van Ginneken. Toward automated segmentation of the pathological lung in CT. IEEE Transactions on Medical Imaging, 24(8):1025-1038, Agosto 2005.

[31] J. Tschirren, T. Yavarna, and J.M. Reinhardt. 3D segmentation of the airway tree using a morphology based method. $I J$ CAI 2009 Workshop on Explanation Aware Computing, pages 227-238, 2009.

[32] WHO. Global strategy for the diagnosis, management and prevention chronic obstrutive pulmonar disease. Technical report, World Health Organization, 2014.

[33] Geórgia Freire Paiva Winkeler. Acute effects of administration of continuous positive airway pressure mode not about invasive pulmonary parenchyma of healthy volunteers in supine and prone positions: Changes in high resolution computed tomography. Master's thesis, Department of Clinical Medicine, Federal University of Cear, Fortaleza, 2006.

[34] X.C. Yin, C.P. Liu, and Z. Han. Feature combination using boosting. Pattern Recognition Letters, 25:2195-2205, April 2005 . 